\title{
DIE NATURWISSENSCHAFTEN
}

WOCHENSCHRIFT FUR DIE FORTSCHRITE DER NATURWISSENSCHAFT, DER MEDIZIN UND DER TECHNIK

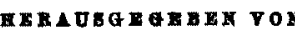

DR. ARNOLD BERLINER UID PROF. DR AUGUST PÜTTER

Siebenter Jahrgang.

18. Juli 1919.

Heft 29.

\section{Über die Modifikationen kristalliner Stoffe.}

Von Geh. Reg.-Rat Dr. F. Rinne, P'rofessor an der Universität Leipzig.

1. Leptonischè Metamonphosenreihe der Materie. Durch den Wechsel des. Wärmegrades der Materie, d. h. durch Veränderung der Beweglichkeit ihrer Leptonen oder Feinbauelemente ist es, wie allbekannt, möglich, Stoffe die lange Reihe won Metamorphosen durchlaufen zu lassen, die sich über den gasigen und flüssigen sowie kristallin-festen Zustand hinerstreckt. Eine solche Reihe setzt sich aus Strecken stetiger feinbaulicher Anderung und trennenden Sprüngen zusammen. Im gasigen Zustande der Dinge hat man es mit wirr duxcheinander ,nomadisierenden", voneinander praktisch unabhängigen Leptonen in Atomoder Molekélform zu tun. Beim Übergang zur Flüssigkeit treten sie durch lose von einem zum anderen Individuum sich schlingende Kraftlinien in lockeren Zusammenhang, beim Kristallisieren erfolgt eine dreidimensional periodische Ordnung
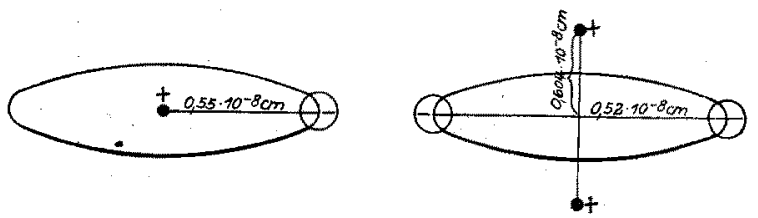

Fig. 1. Wasserstoffatom und Wasserstofimolekul.

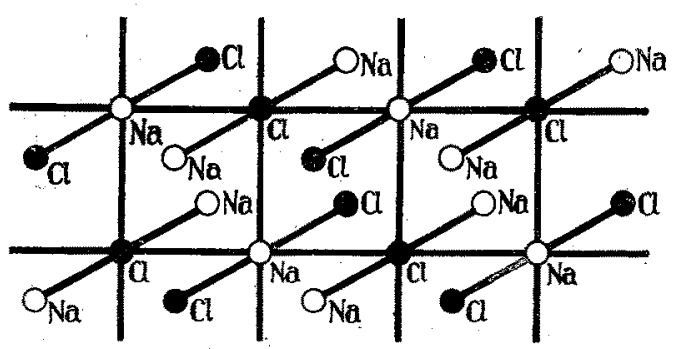

Fig. 2. Leptonistischer Bau des Steinsalzes.

der Atome oder Atomgruppen: das Raumgitterprinzip wird dem Aggregat der Teilchen ubergeprägt. Und wie man z. B. nach der Vereinigung zweier Wasserstoffatome zu einem Molekül nicht mehr erkennen kann, zu welchem Atomkern ein bestimmtes Elektron gehörte, so ist es im Kristall, als dem neuen chemischen System, im allgemeinen auch nicht mehr bekundbar, welche Atome, z. B. im NaCl-Steinsalzbau der Fig. 2, einst ein Molekül miteinander bildeten. Die chemischen Hauptbindungen schneiden im Kristall nicht mehr, wie bei Gasen und Flüssigkeiten an Molekelgrenzen $a b$. sie durchziehen vielmehr das ganze Gebilde in nach Abstand und Richtung gleichmäBiger Verkettung. Hinzugekommen ist als Gegensatz zum. Molekelcharakter die Fähigkeit, durch Wachstum dem Kristall neue Teilchen in weitergreifender atomistischer Verbindung anzugliedern, ohne den chemischen Charakter des Stoffes zu ändern. Das Gewichtsverhältnis der Stoffe ist das gleiche im Molekül wie im Kristall; das absolute Gewicht ist nur für das Molekül konstant.

Nach all dem kann eine ,Identität der Molekeln" in den verschiedenen Aggregatzuständen nicht anerkannt werden: die Moleküle von Gasen und Flüssigkeiten ändern sich beim Durchlaufen der Metamorphosenreihe periodenweise stetig und sprunghaft; im kristallinen Zustande geht der Molekelcharakter der Materie - vollends verloren. Sehr wohl können sich indes nach dem Akte der Kristallisation noch charakteristische Konstruktionszüge der einstigen Molekeln herausheben, etwa im Sinne von $P$. v. Groth Ringbildungen, auch ionenartige Komplexe; sie sind gewissermaßen geometrische Radikale (oder Leptyle, wie man sie in Analogie zu den chemischen Radikalen nennen könnte). Die Baugruppen $\mathrm{Ca}$ und $\mathrm{CO}_{3}$ beim Kalkspat, die $\mathrm{TiO}_{2}$-Knäuel in der -Struktur von Rutil und Anatas sind Beispiele dafür.

\section{Modifitationsfaktoren.}

AuBer den sprunghaften Wandlungen rom Zustande eines Gases in den einer Flüssigkeit und von diesem zum kristallinen Körper sind Untersprünge im jeweiligen Bereiche dieser "Aggregatzustände" möglich; von ihnen seien hier die im kristallinen Material als "Modifikationsänderungen" sich vollziehenden insbesondere gewürdigt. Vorweg ist $z u$ vermerken, daß die.Modifikationen eines Stoffes aber nicht immer in einem solchen epigenetisch engen Verhältnis der Ineinanderverwandelbarkeit durch Wechsel der Temperatur stehen, daß vielmehr manche weniger abhängig davön erscheinen. $\mathrm{Ob}$ die eine oder eine andere Modifikation sich bildet, hängt eben oft nicht nur vom Wärmegrad coder rom Druck bzw einer Kombination dieser beiden Umstände) ab, sondern auch von dem, was man ,stoffliches Feld" nennen känn²). Wie im großen

1) Eine Metamorphose, die im kritischen Zustande stetig vollzogen wird.

2) Bereits $F$. Grandjean wandte den entsprechenden anschaulichen Namen, champ moléculaire de contact" an. Im Anschlus daran nahm ihn $R$. Gro $\beta$ bei Kristallisationsstudien unter der Bezeichnung molekulares bzw. Atomfeld auf. 
Betriebe der Natur die Sterne aufeinander wirken, so werden auch im Mikrokosmos des Moleküls und des Kristalls die Konstellation und die Bewegung der Teilchen von der Umgebung der Partikel in Nahewirkung beeinfluBt. Die molekularen Vorformen der Kristallisation und damit letztere selbēr hängen von den stofflichen Genossen ab. So wird die Herausbildung von Kalkspat oder Aragonit aus einer calciumkarbonathaltigen wässrigen Lösung dadurch bedingt, ob sie rein ist oder Magnesiumsulfat führt, ob also das chemische Feld lediglich aus Karbonat und Wasserteilchen besteht oder sich Sulfatleptonen ihm hinzugesellen; bei dem für diese Verhältnisse besonders zur Demonstration geeigneten Beispiele des sauren Phenylakridoniumsulfats hängt es entsprechend von dem Gehalt an Alkohol, Wasser und Schwefelsäure $a b$, ob ein rotes monoklines oder grünes triklines "Isomeres" entsteht.

\section{Morphologie der Kohlenstoffmodifitationen.}

Bei den Modifikationen, die sich beim Wechsel der Temperatur: ineinander umwandeln, ist die äuBere Deformierung des Kristallbaus gelegentlich so gering, daB die Metamorphose sich ohne Zerfall der Gestalt vollzieht. Das läßt sich z. B. beim Borazit oder Quarz sehr gut beobachten, bei denen es sich beim Umschlag nur um Minuten betragende Winkeländerungen handelt. Von Interesse ist es, daß beim Erhitzen, insbesondere im Quarz, dicht vor der Modifikationswandlung die geometrischen und optischen Veränderungen sich auffallend kräftig abspielen, was auf eine Ansammlung von feinbaulicher Spannung vor der sprunghaften Anderung als Auslösung dieser Spannung hinweist. Bei manchen anderen Stoffen kommt es dabei zum äuBeren Zusammenbruch des Kristallgebäudes, so bei der Umänderung von Diamant in Graphit. Die nähere Untersuchung zeigt indes, däB auch bei derartigen Fällen kristallographische Beziehungen der früheren zu der neu entstandenen Modifikation statthaben können.

In der Hinsicht haben die allgemeinen Verhä]tnisse beim Modifikationswechsel durch die auBerordentlich bedeutsamen röntgenogrammetrischen Untersuchungen und Darlegungen von $P$. Debye und $P$. Scherrer über die beiden Modifikationen des Kohlenstoffes, den Diamanten und den Gràphit, eine besonders lehrhafte Beleuchtung erfahren ${ }^{1}$. Sei es gestattet, hier die Verhältnisse in einfacher Weise zn kennzeichnen. Dem Diamanten kommt nach $W . H$. und $W . L$. Bragg ein würfeliges Raumgitter zu mit C-Atomen an den Ecken und auf den Flächenmitten sowie in den Zentren der abwechselnden Zellen, die man durch die Medianebenen des Würfels in Achtzahl erhâlt (Fig. 3). Miteinander verbunden stellen diese vier inneren Kohlenstoffatome die Ecken eines regelmäBigen Tetraeders dar. Bringt

1) P. Debye und P. Scherrer, Über die Konstitution von Graphit und amorpher Kohle. Physikalicehe Zeitschr. Bd. 18 , S. $291,1917$. man einen solchen stereochemischen Diamantkörper mit einer seiner Diagonalen von Ecke zu Ecke in vertikale Stellung, so erscheint er als Rhomboeder und das vorhin erwäbnte Tetraeder als trigonale Pyramide mit der Grundfläche (Fig. 4 links). Den Graphitbau erhält man nach Debye und Scherrer durch Dilatation des Diamantmodells in Richtung der besagten Körperdiagonale von $6,12 \cdot 10^{-\mathrm{s}}$ auf $10,23 \cdot 10^{-8} \mathrm{~cm}$. Die horizontalen Ausmase bleiben dieselben. Wie am Elementarwürfel des Diamanten liegen die C-Atome des Graphits an den Ecken und auf den Flächenmitten des nun sehlank rhomboedrischen Elementarparallelepipeds; die innere, beim Diamanten tetraedrisch-trigonale Pyramide ist zu einer steileren trigonalen Pyramide geworden, und ihre Grundfläche und Spitze lagern

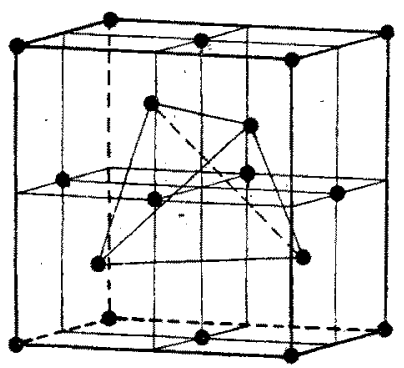

Fig. 3. Elementarkörper des Diamanten.

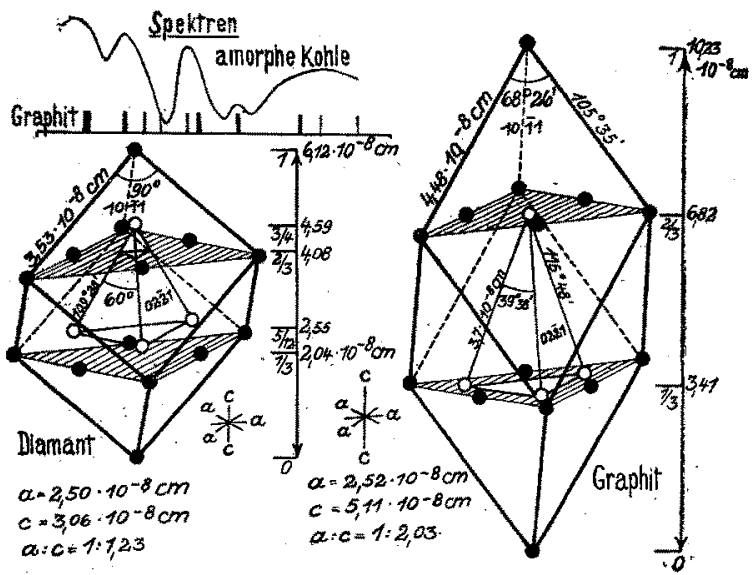

Fig. 4. Vergleich des Feinbaus yon Diamant und Graphit.

runmehr in den Ebenen, die man durch die Atome auf den Flächenmitten legen kann. In Fig. 4 sind eine Anzahl von AusmaBen für den näherinteressierten Leser eingetragen.

\section{Stereochemie der Kohlenstoffmodificationen.}

Wie Debye and Scherrer bereits heraushoben, liegen im Diamant- und Graphitbau die Grundlagen der ehemischen Verkettung von $\mathrm{C}$-Atomen vor. Auch diese Umstände in Zeichnungen in besonders einfacher / Weise herauszuheben, sei hier gestattet. Fig. 5 stellt einen (mit Fig. 3 und 4 nicht zusammenfallenden) Ausschnitt der Diamantstruktur vor. Man erkennt in der Mitte ein-C-Atom, um welches herum sich vier 
andere Atome an die Ecken eines regelmäBigen Tetraeders gestellt haben. Jede dieser Ecken ist wiederum der Mittelpunkt eines Tetraeders, und so sprossen gewissermaßen die chemischen Tensoren durch das ganze Bausystem des Diamanten gleichmäBig fort. Ist also ein Diamantkristall der Repräsentant der aliphatischen Verknüpfung, so tritt nach Debye und Scherrer im Graphit die aromatische Ringbildung deutlich hervor. Er besteht aus Tafeln. mit Sechserringen, die, wie Fig. 6 zeigt, gegeneinander verschoben sind. Damit ergeben sich Bindungen von $1,45.10^{-8} \mathrm{~cm}$

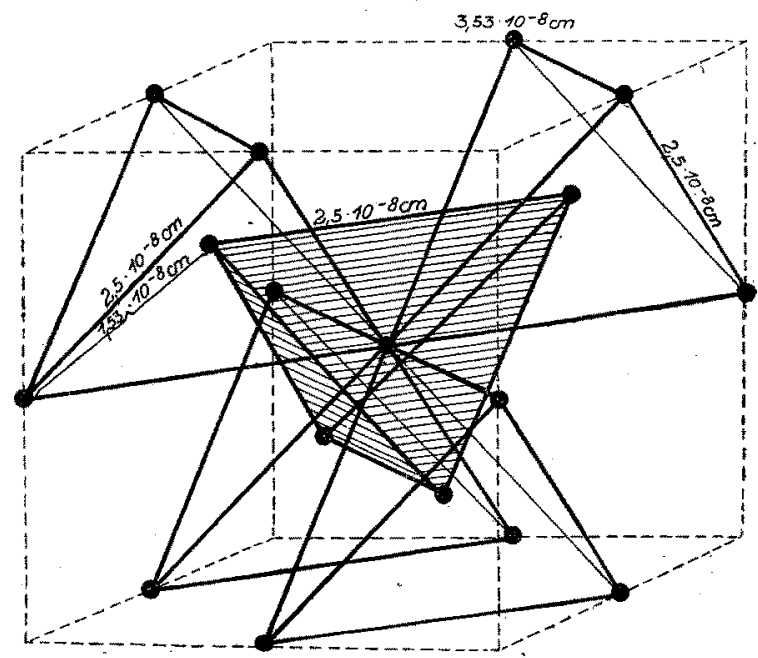

Fig. 5. Stereochemie des Diamanten.

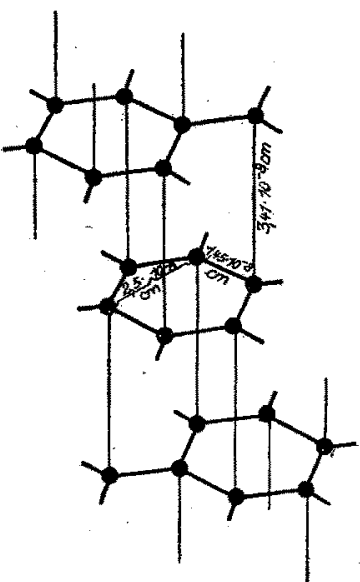

Fig. 6. Stereochemie des Graphits.

Länge in der Tafelebene und solche mit $3,41 \cdot 10^{-8} \mathrm{~cm}$ nach oben und unten in abwechseInder Orientierung.

Ws ist von Interesse, daB man die Bautypen ron Diamant und Graphit noch in anderer Beziehung verwandtschaftlich nebeneinander stellen kann, wie ich es in Fig. 7 and 8 zeigen möchte. Sowohl Diamant als auch Graphit läßt sich aus parallelen Sechserringen aufbauen, die jeweils die obere und untere Fläche eines hexagonalen Prismas ausmachen. Die Höhe dieser Prismen beträgt beim Diamanten nur 1,53, beim Graphit $3,41 \cdot 10^{-8} \mathrm{~cm}$; sie sind beidemal mit ihrer Achse gegeneinander um $1,45 \cdot 10^{-8} \mathrm{~cm}$ entsprechend den Figuren verschoben. Beim Diamanten haben sie einen Abstand von $0,51 \cdot 10^{-8} \mathrm{~cm}$, während sie beim Graphit aufeinander liegen, so daB also die Oberseite eines unteren Prismas mit der Unterseite des nächst höheren in eine Ebene kommt.

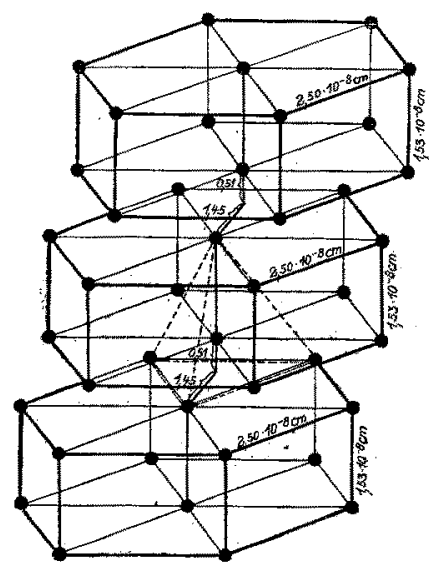

Fig. 7. Feinbau des Diamanten.

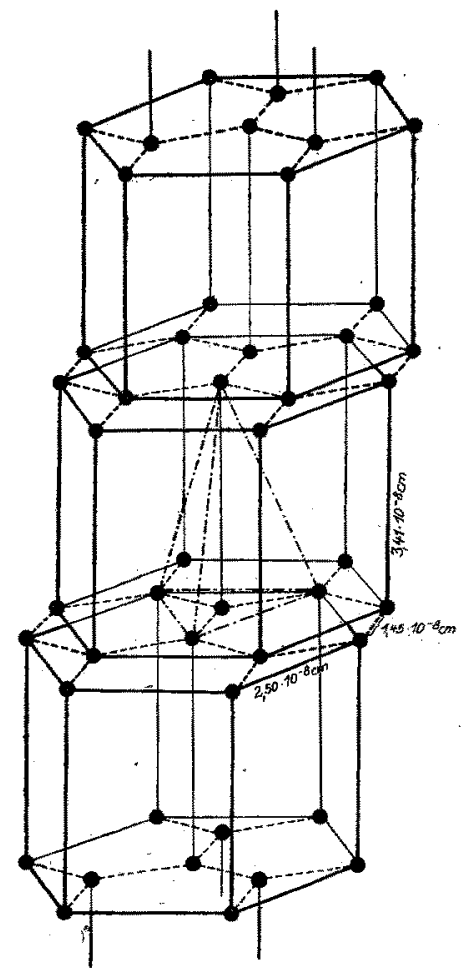

Fig. 8. Feinban des Graphits.

Als Modell mit nach den Vertikalen verschiebbaren Sechserringen gebaut, kann dies einfache Strukturschema des Diamanten in das des Graphits und umgekehrt leicht übergeführt werden. Wie in Fig. 4 heben sich die Beziehungen der Bauart von Diamant und Graphit in den Fig. 7/8 anschaulich heraus, insbesondere auch beim 
Voumvergleich die Verschiedenheit der ,Konzentration" des Kohlenstoffs in den beiden Modifikationen. Die Richtungsbeziehung der Fig. 7/8 zu den Abbildungen Fig. 4 ergibt sich bei Beachtung der eingezeichneten Tetraeder bzw. trigonalen Pyramiden ohne weiteres.

\section{Moditikationsisotypie.}

Sei es nun gestattet, in Ansehung des jetzt so außerordentlich anschaulichen Vorganges der Verwandlung des Diamanten in Graphit einige Betrachtungen allgemeinerer Art anzustellen.

Die naturkundiche Bedeutung der Erscheinung liegt wesentlich darin, daß sie ein experimentell gefestigtes. Beispiel für die Anschauung ist, nach der die verschiedene Konstellation der nämlichen Atome auch in dem Kristall genannten chemischen System bedeutende Wandlungen in geometrischer, physikalischer und chemischer Hinsicht mit sich bringt. In der Erkenntnis wird man also nun weiter versuchen, die grundlegenden Gesetzmäßigkeiten solcher Waudlungen herauszuárbeiten. Dabei liegt es wohl am nächsten, die morphologischen Motive zu erfassen, wie es in folgender Uberlegung angestrebt sei.

Anschaulich entwickelt sich durch die Schem mata der Fig. 4 die Deformation der Diamantzur Graphitform. Man wird sich vorstellen müssén, daß beide Architekturen standhafte Bautypen präsentieren. Natürlich werden die Architekturen bis zu einem gewissen MaBe von der besonderen: stofflichen Art der Substanz, im vorliegenden Beispiel des Kohlenstoffs, abhängen. Von vornherein ist es aber wahrscheinlich, dab sich in ihnen und entsprechend bei den Modifikationen anderer Stoffe geometrische Stabilitätstypen kenntlich machen. Hält man in dem Sinne Umschau unter den Kristallformen sonst bekannt gewordener Fälle, so heben sich in der Tat beim Uberblick besonders der chemisch einfach aufgebauten Stoffe, solche Typen stabiler Bauart deutlich heraus, eine Erscheinung, die ich bereits fruher Isotypie genanut habe. Fin weitverbreiteter Modifikationstypus ist der isometrische; ihm gehört auch der Diamant an. Stellt man ihn trigonal wie in Fig. 4 auf, so kennzeichnet sich seine bei allen isometrischen Stoffen gleiche Winkelart durch das Achsenverhaltnis $a: c=1: 1,23$. AnBerst verbreitet, und zwar gleichfalls unabhängig von der jeweiligen Zusammensetzung, ist weiterhin ein hexagonaler Modifikationsbautypus mit $a: c=1: 1,64$. Dazu gesellt sich nun der Graphitbau mit $a: c=$ 1:2,03. Man erkennt, daß sich bei diesen drei Typen die Abschnitte auf der vertikalen Banrichtung $c$ wie $3: 4: 5$ verhalten, ein einfaches, in sich harmonierendes MaBverhältnis, das diese drei Typenformen chemisch einfach zusammengesetzter Stoffe miteinander verknüpft. Die von der jeweiligen chemischen Art abhängigen spezifischen Abweichungen, wie sie sich beim hexagonalen Typus zufolge der großen Zahl seiner
Glieder verfolgen lassen, ist gering. Z. B. sind kennzeichnende Kristallwinkel für einige seiner Angehörigen folgende: $\mathbf{M g} 62^{\circ} 9^{\prime}$; ( $\left.\mathrm{Ir}, \mathrm{O}_{\mathrm{s}}\right) 62^{\circ} 0^{\prime} ; \mathrm{ZnO} 61^{\circ} 54^{\prime} ; \mathrm{ZnS} 61^{\circ} 35^{\prime}$; Ag.J $62^{\circ} 9^{\prime} ; \mathrm{CSi} 62^{\circ} 9^{\prime} ; \mathrm{H}_{2} \mathrm{O} 61^{\circ} 50^{\prime} ; \mathrm{SiO}_{2}$ (Tri. dymit) $62^{\circ} 21^{\prime}$ usw. Die Ahnlichkeit bei chemischer starker Verschiedenheit ist nicht zu verkennen 1 ).

\section{Atomvolumverhälnisse der Modifikation.}

Bezüglich der morphologischen Verhältnisse der Modifikationen kristalliner Aggregationen jst weiterhin von Interesse, die Atomvolumina zu verfolgen. Zwar läßt sich die Dimensionierung der Atome mit ihrem Atomkern und elektronischen Trabanten in den verschiedenen Richtungen aus den rö̀tgenogrammetrischen Ergebnissen an Kristallen noch nicht erkennen, nimmt man indes vorerst die Atomvolumina als kugelförmig an, so wäre ihr Maximalradius e gegeben durch den kleinsten Abstand zweier gleicher Atome im Kristallbau. Es interessiert, zu wissen, ob diese Distanz bei den Modifikationen eines Stoffes gleich ist oder nicht. In der. Hinsicht findet man beim Diamant $\varrho=1,53 \cdot 10^{-\mathrm{s}} \mathrm{cm}$, beim Graphit 1,45 $10^{-8} \mathrm{~cm}$, also ähnliche MaBe. Daß aber der unterschiedliche Wert kennzeichnend ist, ersieht man aus den entsprechenden Zahlen für Titan. Aus den von Vegard für Rutil und Anatas (beide $\mathrm{TiO}_{2}$ ) gegebenen Daten findet man für $\mathrm{a}$ des Titanatoms 3,51 bzw. $3,0^{-10^{-8}} \mathrm{~cm}$, also deutliche Differenzen ${ }^{2}$ ).

\section{Physikalische und chemische Stereometrie der Modifitationen.}

Für die physikalischen Verhältnisse der $K_{0-}$ häsion und die Umstände des chemischen Zusammenhaltes ist die Konstellation der. Bauteilchen eines kristallinen Aggregats natürlich gleichfalls von Bedeutung. Es kommt dafür die Anordnung und Dimensionierung der geometrischen Radikale in Betracht, aus denen sich die Modifikationen zusammensetzen. Beim Diamant und Graphit reduzteren sich diese Baugruppen auf Atome. Die Affinitätstensoren von einem zum anderen Kohlenstoffteilchen sind das LängenmaB und zugleich der Anhalt für die Stärke der Bindung; enge Nachbarschaft bedeutet starke physikalischchemische Verkettung, weitere Entfernung schwächere Verknuppfung. In dem Sinne erscheinen die Kohlenstoffatome des Diamanten untereinander gleich stark gebunden, nämlich

1) Auf andere noch bestehende Typen chemisch einfach zusammengesetzter Stoffe sei hier nicht weiter eingegangen. Auch sie werden durch die röntgenogrammetrischen Untersuchungen immer klare herausgearbeitet.

2) Die Volumina der Elementarköroer von Diamant und Graphit sind durch die Debye-Seherresschen Zahlen natürlich ohne weiteres gegeben. Man findet für Diamant $44.10-24 \mathrm{ccm}$ und für Graphit $74,9.10 \mathrm{~cm} \mathrm{ccm}$. Fernerbir hat das. Tetraeder des Diamanten der Fig. 4 einen Inhalt von $1,84,10$ - cem, die entsprechende trigonale Pyramide des Graphits $3,13.10-2 \mathrm{cem}$. 
d'urch Tensoren von $1,53 \cdot 10^{-8} \mathrm{~cm}$ Länge, die des Graphits verschieden stark, insofern hier der Atomabstand in der Endfläche der Prismen von Fig. $81,45 \cdot 10^{-8} \mathrm{~cm}$ beträgt, aber die Entfernung der C-Atome senkrecht dazu $3,41 \cdot 10^{-8} \mathrm{~cm}$ ausmacht, wie das schon $P$. Debye und $P$. Scherrer heraushoben. Solche Umstände der physikalischen und zugleich chemischen Verschiedenheit der Bindungen machen sich nach den Erörterungen von $E$. Schiebold über Kalkspat (Dissertation, Leipzig 1919) deutlich geltend in der Eigenschaft der Spaltbarkeit; sie geht zusammen mit dem Vorhandensein, leptonistisch gedacht, weit klaffender planer Baulücken parallel den Spaltflächen, zugleich aber auch mit dem bedeutsamen Merkmal, daß dementsprechend keine eng zusammengehörigen Baugruppen als geometrischchemische Radikale, mithin keine starken chemischen Bindungen bei der Spaltung zerrissen werden. Die außerordentlich weitgehende Blättrigkeit des Graphits parallel den nur durch schwache chemische Bindung zusammengehaltenen Endflächen der hexagonalen Prismen in Fig. 8 erläutert diese Verhältnisse ganz vortrefflich. Durch die Spaltbarkeit werden die in sich fest gebundenen chemischen Bauteile aus der stereochemischen Formel herauspräpariert.

\section{Allgemeine Morphologie des "Amorphen" und Kristallinen,}

Entsprechend wird man weitere Uberlegungen anstellen müssen über alle sonstigen Eigenschaften der Modifikationen, um sie aus der Mechanik der kristallinen Systeme abzuleiten, im Falle des Diamanten und Graphits also z. B. uber die große Härte, klare Durchsichtigkeit und chemische Widerstandsfähigkeit des einen und über die, demgegenüber so auffällige, außerordentliche Milde, die völlige Lichtundurchlässigkeit und die Oxydierbarkeit des anderen Isomeren; ein noch weites Feld allgemein bedeutsamer Forschung.

Man ist am Anfange des Weges. Jedoch eröffnen sich schon manche physikalisch-chemische Ausblicke in die Weite. Sei in der Hinsicht bei der vorliegenden Betrachtung an Hand des Beispiels Diamant und Graphit, zu denen sich noch die sogenanate amorphe Kohle gesellt, der Gedanke verfolgt, wie man sich im Sinne der Lepto'ogie oder Feinbaulehre das allgemeine Verhaltnis der amorphen $z u$ den Kristallinen Stoffen, die man. ja als Modifikationen einer Substanz auffabt, ausmalen kann. In der Hinsicht ist zunächst darauf zu verweisen, daß die Individuen des amorphen Zustandes, die Atome, Ionen und Molekeln, an sich ebensowenig, gestaltlos"s und ebensowenig innerlich regellos sind, wie die Einheit des dreidimensional periodischen Zustandes, der Kristall. Man kann auf sie die Symmetrieforschungen bezüglich Inversion, einfache und Schraubenachsen, gewöhnliche und Gleitspiegelebenen und dèr Kombination dieser Symmetrie-

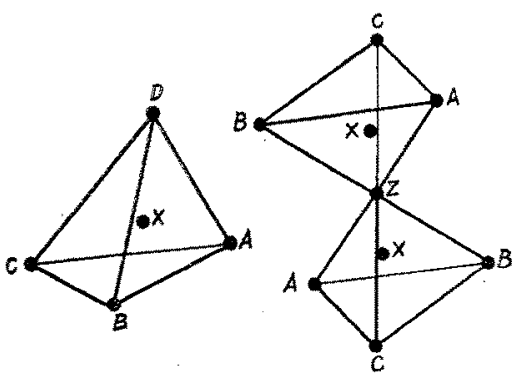

$a$

b
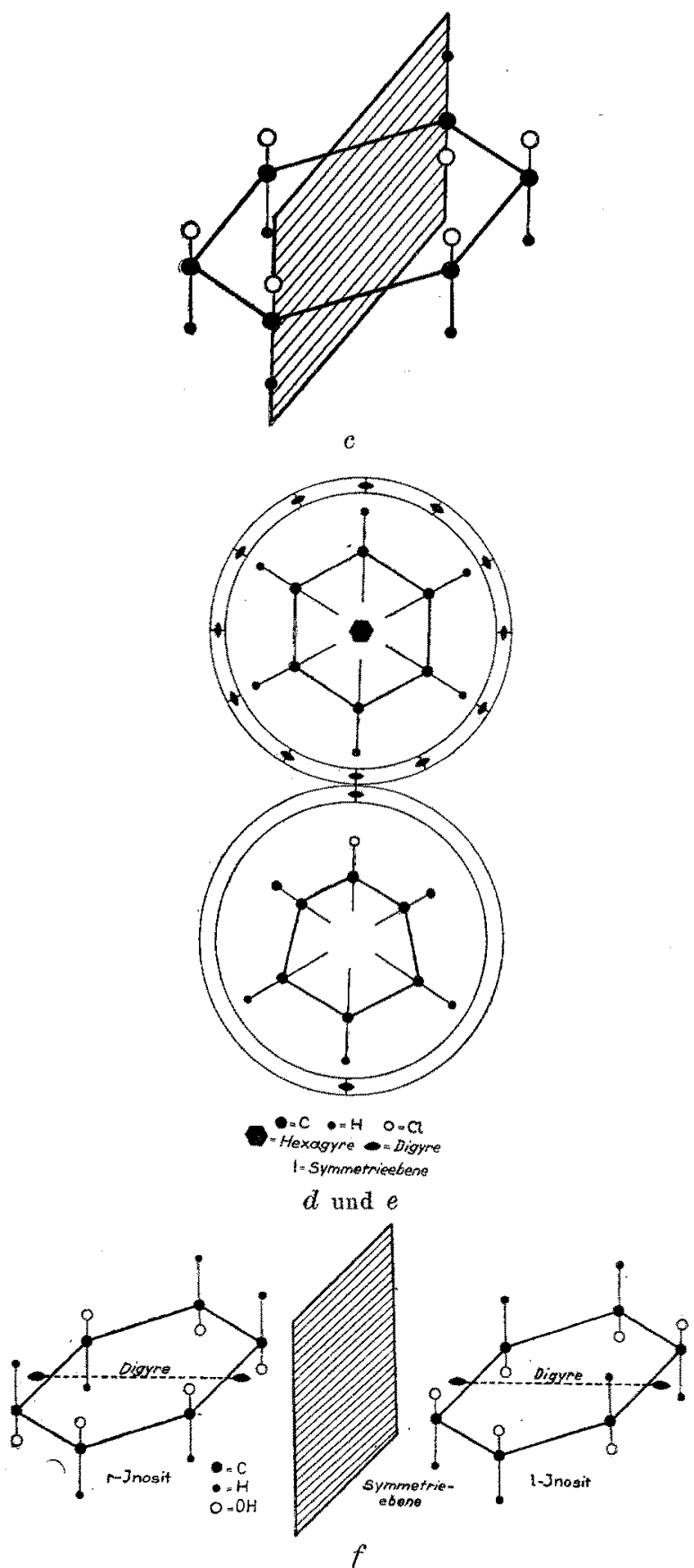

Fig. 9. Symmetrieverhältnisse von Molekülen. 
elemente ${ }^{1}$ mit demselben Rechte, wenn auch noch nicht mit derselben Leichtigkeit des Erfolges, anwenden, wie es bei den Kristallen hinsichtlich ihres Aussehens und Feinbaues geschieht ${ }^{2}$ ). Die Fig. 9 gibt einige Beispiele. Das allgemeine Interesse bezüglich der Geometrie solcher Gebilde besteht darin, daB sie den Generalfall der Feinbaustrulituren vorstellen: die Beschränkung des Baurhythmus auf die 2-, 3-, 4- und 6-7ahl, wie sie durch das kristallographische Grundgesetz gegeben ist, fält bei ihnen fort.

So können also die Symmetriestudien bei den leptonistischen Bildungen in erweiterter Form angesetzt werden; es ist zu erwarten, daß sich aus den bereits vorhandenen Ansätzen das Gegenstück zur Morphologie der Kristalle, eine Leptomorphologie, entwickeln läßt, welche insbesondere auch die Veränderungen behandelt, die sich in derf chemischen Einheiten bei Substitutionen und Isomeriewandlungen einstellen (vergl. z. B. Fig. $9 d, e, f)$, und weiterhin bekundet, welche Beziehungen zwischen diesen beiden Kapiteln der allgemeinen. Morphologie bestehen.

\section{Eigenschaftswechsel der Molekeln mit der Richtung.}

Auch dari man hinsichtlich der Beziehungen zwischen den molekularen Individuen und den Kristallen noch ein zweites Moment annehmen; es ist der manchmal mit Unrecht für den kristallinen Zustand reservierte gesetzmäBige Eigenschaftswechsel mit der Richtung. Er bekundet sich auch bei molekularen Gebilden ja schon in den. morphologischen Schematen, und hinsichtlich der physikalischen Verhältnisse tritt er bei Gasen, Flüssigkeiten und Schmelzen nur deshalb nicht heraus, weil die Molekeln bei thnen wirr durcheinanderliegen und als Gesamtheit nach allen Richtungen gleiche Mittelwerte, liefern. Im Falle der Parallelisierung einer Baurichtung. der Individuen bekundet sich in günstigen Umständen der molekulare Richtungssinn in den optischen Eigenschaften, so ev. im Falle der Reibung beim Fließen' des Stoffes und insbesondere unter dem Einflusse eines elektrischen oder chemischen Feldes. Für elektrische Zwangsstellung der Molekeln ist das Nitrobenzol ein bekanntes Demonstrationsobjekt; für den Fall gegenşeitiger Molekelorientierung unter der Wirkung ihrer eigenen Kraftlinien sind ,flussige Kristalle" bedeutsame Beispiele, In stofflicher Nahewirkung aufeinander gruppieren sich deren Moleküle mit einer ausgezeichneten Richtung auf gröbere Bezirke parallels), sie be-

1) Vergl. z. B. $F$. Rimne, Zur ältesten und neusten Kristallographie. Diese Zeitsehr. 1916, Heft $17 / 18$.

2) Séi in der Hinsicht der Darlegungen von $F, M$. Jaeger, Lectures on the principle of symmetry, Amsterdam 1917, gedacht.

3) Man muß bederiken, daB die Wellenlänge gewöhnlichen Lichtes gegenüber den Molekeldimensionen eine ungeschlachtete Größe ist, im Verhältnis etwa. wie die Länge eines Hauses zu der einer Streichholz- kunden dann ihren molekularen optischen Richtungssinn nach Art optisch einachsiger Kristalle, ohne wahre Kristalle vorzustellen; es fehlt ihnen dazu die Raumgitterstruktur. Die allgemeine Eigenschaft der Molekeln, richtungsverschieden zu sein; die in optischer Hinsicht für gewöhnlich durch wirre Lagerung kompensiert ist, findet bei den ,flussigen Kristallen" ihren Ausdruck zufolge der Leichtigkeit, mit der ihre Molekeln sich mehr oder minder vollkommen zueinander auf größere Strecken parallel richten. Die Besonderheit der flüssigen Kristalle gegenüber anderen Atomaggregaten liegt also nur in der extremen (nach Vorländer langgestreckten) einer Parallelrichtung günstigen Bauart ihrer molekularen Individuen begründet; sie gehören in ihren typischen Beispielen der Molekularchemie, nicht der Kristallchemie an, und sind als ,Fastkristalle" in ihrem selbständigen Bestreben zur Parallelisierung höchst interessante Übergangsformen zwischen amorpher und kristalliner Materie, und dann vom nämlichen Charakter wie das Nitrobenzol in seiner elektrischen Zwangsorientierung.

\section{0." Morphologische Ubbrgänge vom Kristallinen zum Amorphen.}

Weiterhin zeigen nun Röntgenuntersuchungen nach dem Verfahren von Debye und Scherrer gleichfalls, dab zwischen, amorphem" und kristallinem Material geometrische Verwandtschaftsbeziehungen und Ubergänge vorhanden sind. Für das einschlägige Experiment ist kennzeichnend, daB auch die kristallinen Partikel in wirrer Lagerung, nämlich als Teilchen eines sehr feinen Pulvers; benutzt werden. So kommen dann Reflexkegel durch Spiegelung an den feinbaulichen durch die Atome gelegten Ebenen rund um den

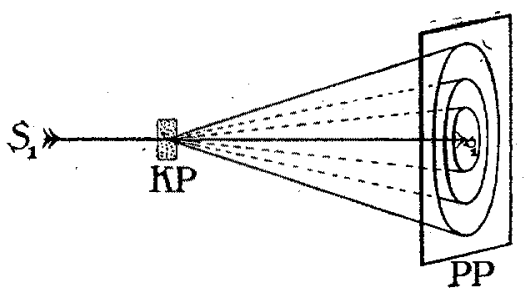

Eig. 10.

Primärstrahl zustande. Ein Molekelgemisch läßt hierbei nur verwaschene ineinander verklingende Strahlenkegel erkennen, wie das die Intensitätskurve für amorphen Kohlenstoff in Fig. 4 zeigt; seine reflektierenden Ebenen sind weit lockerer besetzt als im kristallinen Pulver des Graphits, dessen scharfe Reflexe auf der Wagerechten oben in Fig. 4 unter der Intensitätskurve der Beugung: amorphen Kohlenstoffs nach Debye und Scherrer gezeichnet ist. Es herrschen, wie man unmittel-

achachtel. Um ihre Doppelbrechung zu bekunden, müssen sehr viele dieser Molekelschachteln mit einer Richtung parallelisiert werden. 
bar erkennt, hierbei im Grunde nur Unterschiede der Schärfe des Beugungsexperiments, micht Wesensverschiedenheiten aufdeckende Gegensätze. Beim ,amorphen" Kohlenstoff wie bei anderen amorphen Substanzen verhalten sich die Molekeln wie feinbauliche Fetzen von Kristallen. In je feinere Teile letztere dispergiert sind, um so ähnlicher werden sie dem amorphen Material in ibren Beugungserscheinungen und ihren anderen Eigenschaften. Im Sinne der Kolloidchemie wird es auch hier alle Übergänge im Zerteilungsvorgange der Materie geben. Beim Aggregationsbestreben bilden die A tomkomplexe unter dem Einfluß ihrer Umgebung zunächst molekulare Vorformen, und beim Akte der Kristallisation selber fügen sich die Atome in gegenseitiger Nahewirkung durch das ganze neue System hindurch zum Punktsystem zusammen. Wenin auch starke Konstruktionslinien der Molekeln bei der Bildung und beim Wachsen der Keime übernommen werden, so machen sich doch dabei auch néue, durch die Einheit des Kristalls gleichmäBig sich er streckende Affinitätsverknüpfungen geltend. Die Auffassung einer rein physikalischen Aneinanderlagerung paBt nicht recht in den Vorstellungskreis der Feinbaulehre.

\section{Physikalische Isomerie.}

Damit verändert sich auch die Erläuterung der ,physikalischen Isomerie", wonach es sich bei ihren Modifikationen um die verschiedene Gruppierung gleicher Molekel handeln sollte; in Wirklichkeit werden auch hier die isomeren Gegensätze auf stereochemischen Unterschieden beruhen. Man wird also bei der Unterscheidung ron chemischer und physikalischer Tsomerie auf ein anderes, schon immer herangezogenes Moment Nacharuck legen, nach welchem für physikalische Isomerie kennzeichnend ist, dab sie in der Kristallisation ersteht und mit der Amorphosierung, als Zerteilung in die für beide Modifikationen gleichartigen Molekel wieder vergeht, während chemische Isomerie atis dem molekularen; also gasigen oder flüssigen Zustande der Stoffe in den kristallinen Bau übernommen wird, und bei dessen Molekularisierung nicht verschwindet; die Isomerie besteht hier vielmehr in Form zweierlei Molekel fort, die genetisch je mit einer kristallinen Modifikation verbunden sind. Physikalische Isomerie ist also Kristallisationsisomerie, aber gleich chemischer Isomerie stereochemischer Art.

\section{Sammelkristallisation.}

Ein besonders merkwürdiger hien noch zu erwähnender Fall der in Rede stehenden Aggregationsvorgänge ist die Vereinigung kleiner, bereits festkristalliner Partikel derselben Modifikation zu einheitlichen größeren Kristallen, eine Erscheinung isophaser Kristallisation, die sieh in dem ,Finformen" bei Metallen, der Marmorisierung von Kalkstein, dem Körnigwerden von Eis, der Vereinheitlichung von
Wolframpulver zu viele Zentimeter langen Kristallen der Glühlampen und in anderen Fällen zeigt. Das Kennzeichnende dabei ist, dab es sich um einen besonderen Akt des Wachsens durch Kristallisieren, d. h. der gleichmäBigen Fortführung eines regelmäBigen Punktsystems handelt. Daher empfiehlt es sich, den anschaulichen Namen Sammelleristallisation zu gebrauchen. Das Kristallwachstum ist also nicht auf die Angliederung und den Einbau der Vorformen von Gasen, Tösungen oder Schmelzen-beschränkt. Der Einflub kristalliner Teilchen erstreckt sich über die Individuumsgrenze auf andere bereits kristalline Partikel, und die Kraftlinien, welche von den durch Größe bevorzugten Körpern ausgehen, drängen kristalline Nachbarteilchen ,in Parallelstellung und ordnen deren Raumgitter um* ${ }^{* 1}$ ).

\section{Besprechungen.}

Bloch, W., Einführung in die Relativitätstheorie. Aus Natur und Geisteswelt Bd. 618. Leipzig and Berlin, B. G. Teubner, 1918, $100 \mathrm{~S}$. und 16. Fig. Preis M. 1,20 .

In den 14 Jahren, die seit der Schöpfung der Relativitätstheorie durch Albert Einstein verflossen sind, ist eine beträchtliche Zahl von Vorträgen und Bro. schüren erschienen, die in mehr oder weniger popu. litrer Darstellung den naturwissenechaftlich inter. essierten Laien und den Studierenden auf die Höhen der Einsteinschen Gedankenwelt führen sollen. Unter den mir bekannten "Einfuhrungen" dieser Art halte ich die vorliegende kleine Schrift vón Bloch für die beste. Hier ist formell und inhaltlich die Klarheit erreicht, die zur Bewältigung eines so schwierigen und reizvollen Stoffes nötig ist. Ohne ein Zuviel an mathematischem Rüstzeug, aber auch ohne eine gar zu üngstliche Scheu vor der Formel, die ja für den, der sie zu lesen versteht, den prägnantesten Ausdruck gewisser Tatsachen darstellt . wird hier der Ẃeg beschritten, der aus den Niederungen zu dem Gipfel der Relativi? tätserkenntais fiuhrt.

Daß eine gute Darstellung der Relativitätstheorie selbst fiir den. Kenner; und um so viel mehr eine belehrende Einführung für den Nichtwissenden keine leichte Aufgabe ist, das weiß jeder, der in mühsamern Ringen mit den zähen Vorurtellen der Tradition sich zu der Klarheit der Einsteinschen Raum-Zeit-Auffassung durchzuarbeiten versucht hat. Überall liegen Steine des Anstobes am Wege, an denen sich der an iberlieferte Formen gewöhnte Geist wundstöBt. Auf Schritt und Tritt tauchen Finwände auf, denen begegnet werden muB, $Z$ weitel, die zu zerstreuen sind. Es ist ein Dornenweg, aber 'der Ausblick von der Möhe ist weit und umfassend und belohnt die Mühen des. Weges in reicher Maße,

Der Inhalt des kleinen Buches sẹi hier kurz skizziert:

Nach einem einleitenden Kapitel, in dem, die Begriffe der üblichen Raum- und Zeitmessung ausein. andergesetzt werden, wird der Leser mit dem Galileischen Relativitatsporinzip der Mechanik bekannt gemacht, das die mechanische Gleichwertigkeit aller gleichförmig geradlinig gegeneinander bewegten Be-

1) Vergl. F. Rinne, Bd. Chemie der Kultur der Gegenwart, 1913. 